This item was submitted to Loughborough's Research Repository by the author.

Items in Figshare are protected by copyright, with all rights reserved, unless otherwise indicated.

\title{
Pulsed laser deposition of metallic multilayers: the influence of laser power on microstructure
}

PLEASE CITE THE PUBLISHED VERSION

http://dx.doi.org/10.1007/s00339-004-2809-5

PUBLISHER

(C) Springer

VERSION

AM (Accepted Manuscript)

LICENCE

CC BY-NC-ND 4.0

\section{REPOSITORY RECORD}

Al-Busaidy, Mohamed S.K., Olga E. Kusmartseva, and Michael D. Cropper. 2019. "Pulsed Laser Deposition of Metallic Multilayers: The Influence of Laser Power on Microstructure". figshare.

https://hdl.handle.net/2134/10742. 
This item was submitted to Loughborough's Institutional Repository (https://dspace.lboro.ac.uk/) by the author and is made available under the following Creative Commons Licence conditions.

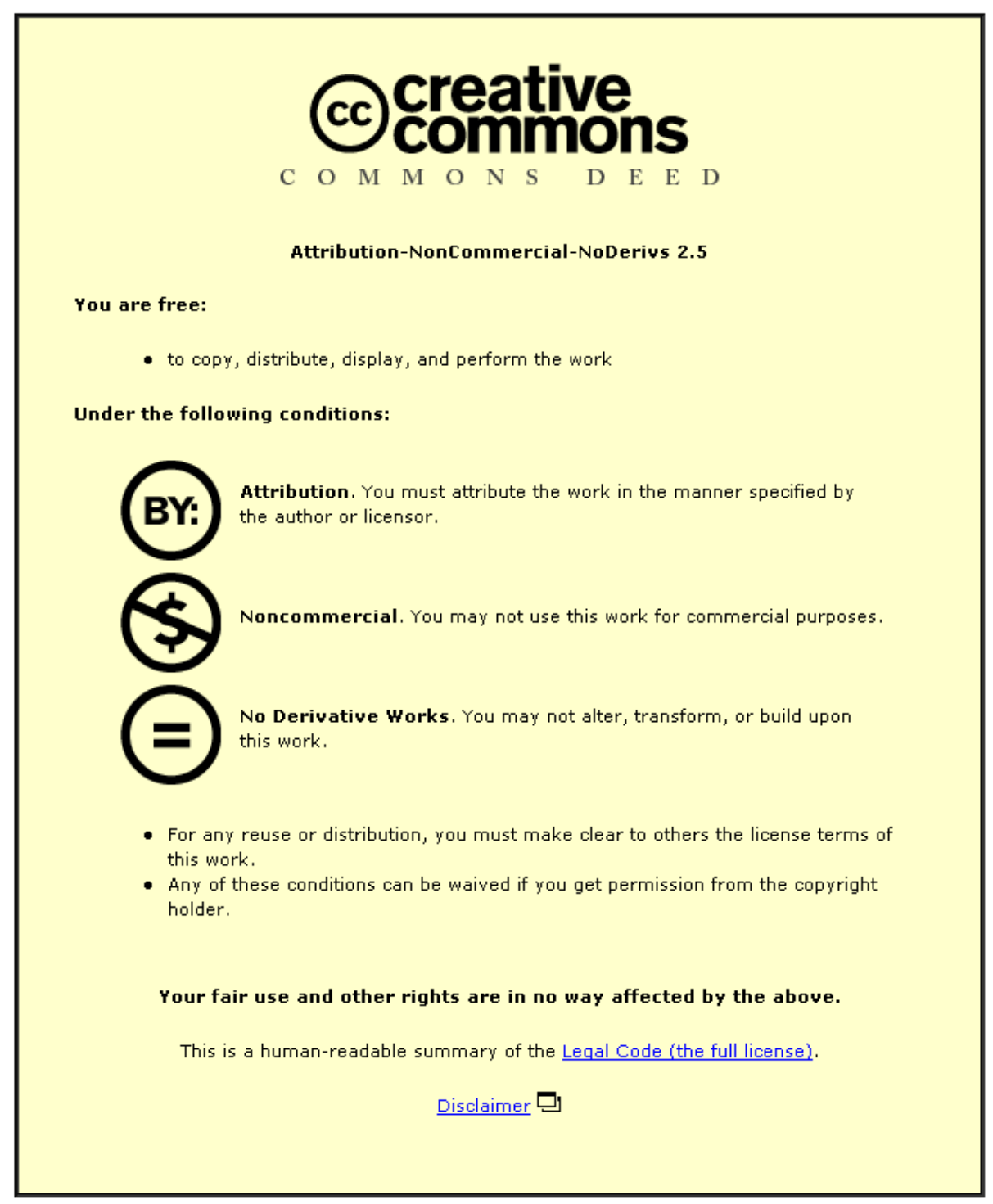

For the full text of this licence, please go to: http://creativecommons.org/licenses/by-nc-nd/2.5/ 


\section{Pulsed LASER Deposition of Metallic Multilayers:}

The Influence of Laser Power on Microstructure

M S Al-Busaidy*, O E Kusmartseva* and M D Crapper*^

"College of Science,

Physics Department,

Sultan Qaboos University,

PO Box 36,

Al-Khod 123,

Sultanate of Oman

'Department of Physics,

Loughborough University,

Loughborough,

LE11 3TU,

UK

Tel: +44 1509223308

Fax: +441509223986

e-mail: m.d.crapper@lboro.ac.uk

^Author to whom correspondence should be addressed.

\section{Abstract}

Multilayers of $\mathrm{Co}$ and $\mathrm{Cu}$ on $\mathrm{Si}(111)$ substrates have been produced by pulsed LASER deposition at the second harmonic of a Nd:YAG LASER (532 $\mathrm{nm}$ ). The effect of varying the LASER power on the film microstructure has been investigated using grazing incidence X-ray reflectivity measurements. Quantitative analysis of the reflectivity curves indicates that higher LASER powers are associated with greater intermixing at the $\mathrm{Co} / \mathrm{Cu}$ interfaces. Offset scans indicate that there is conformal roughness. The deposition process introduces some droplets into the layers, principally of $\mathrm{Cu}$.

PACS: $\quad 61.10 \mathrm{Kw} \quad 68.35 \mathrm{Ct} \quad 68.65 \mathrm{Ac}$ 


\section{Introduction}

The enormous interest in $\mathrm{Co} / \mathrm{Cu}$ multilayers stems from their magnetic and magnetotransport properties $[1,2]$, which have made this system one of the most extensively studied of all the transition metal magnetic multilayers. These properties are now known to depend upon the film microstructure $[3,4]$ and the interface structure of the multilayer $[4,5]$. The micro- and interface structure may be strongly influenced by the growth environment and the deposition technique employed. It has been shown [6] that the utilisation of an energetic growth method, for example plasma assisted d.c. sputtering, can have a strong influence on the roughness of interfaces and surfaces.

Pulsed LASER deposition (PLD) is a widely used method for the deposition of thin films, particularly films of oxides and other compounds and materials that are difficult to deposit by other means. Although not widely used for the deposition of magnetic multilayers, there are many examples of its use in the deposition of multilayers for X-ray optics [7]. However, PLD possesses some attractive qualities that make it potentially useful for the deposition of magnetic multilayer systems. It is, in principle at least, capable of a high degree of control in depositing layers of precise thicknesses. Its ability to provide congruent evaporation could be useful for the newer generation of magnetic ultra-thin film devices that utilise the property of alloys. In addition, the explosive nature of the ablation 
process makes it an energetic deposition technique, with atomic species arriving at the substrate with far more than thermal energy. This energy enhances nucleation and may influence interface smoothness. Its major potential disadvantage is the tendency to produce droplets that, if magnetic, would bridge the required anti-ferromagnetic coupling between successive magnetic layers.

The poor contrast between $\mathrm{Co}$ and $\mathrm{Cu}$ make such multilayers difficult to image in transmission electron microscopy. However, the nature of the layer structure may be examined in detail using grazing incidence X-ray reflectivity (GIXR), where the angles of incidence are between $2^{\circ}$ and $10^{\circ}$ grazing $\left(80^{\circ}-88^{\circ}\right.$ off normal). The geometry makes the method particularly sensitive to surface effects on the nanometer scale and quantitative information on the layer thickness and interface roughness may be obtained by fitting the data computationally. The utility of GIXR is further enhanced by the use of synchrotron radiation, when the tuning of the $\mathrm{X}$-ray energy to the absorption edge enhances elemental contrast by anomalous scattering.

In this paper we describe the application of PLD to the growth of $\mathrm{Co} / \mathrm{Cu}$ multilayers with layer thickness around $20 \mathrm{~nm}$, and in particular the influence of the LASER power on the film microstructure as investigated using GIXR. 


\section{Experimental}

The Loughborough PLD system was developed in a joint project between Loughborough University and Polaron-CVT Ltd, and is based on the design of the system at the IWS Dresden. The LASER is a Nd:YAG with nominal $1 \mathrm{~J} /$ pulse and was operated at $10 \mathrm{~Hz}$. The pulses are focussed into an ion-pumped ultra-high vacuum growth system with a base pressure on $1 \times 10^{-10}$ mbar. The growth system is a typical UHV molecular beam epitaxy system with a preparation chamber and load-lock. The sample stage is equipped with rotation for greater uniformity and heating. PLD targets are incrementally translated under computer control so that successive pulses impinge on adjacent parts of the target and the targets may be exchanged in-situ.

A series of Co/Cu multilayers was deposited using the UHV-PLD system. The Q-switched Nd:YAG LASER was operated in a range of powers between 3.65 and $6.35 \mathrm{~W}$ at the second harmonic of wavelength $532 \mathrm{~nm} .99 .95 \%$ pure elemental targets of $\mathrm{Fe}, \mathrm{Cu}$ and $\mathrm{Co}$ were used and the maximum chamber pressure during deposition was $2 \times 10^{-10} \mathrm{mbar}$. The multilayers were deposited onto an outgassed $\mathrm{Si}(111)$ substrate at room temperature. The nominal structure of the multilayer samples was $\mathrm{Si}(111) / \mathrm{Fe}(\mathrm{x} \AA) /[\mathrm{Co}(\mathrm{x} \AA) / \mathrm{Cu}(\mathrm{x} \AA)]_{18} / \mathrm{Co}(\mathrm{x} \AA) / \mathrm{Fe}(\mathrm{x} \AA)$, with $\mathrm{x}$ close to the second antiferromagnetic maximum at $20 \AA$, which was achieved by varying the number of pulses per layer. The films were then investigated 
using GIXR and scanning Auger microscopy (SAM).

The GIXR measurements were performed on station 2.3 at the SRS [8], Daresbury Laboratory using an X-ray wavelength of $1.3814 \AA$ selected by a monolithic $\mathrm{Si}(111)$ single crystal monochromator. This wavelength is just above the K-absorption edge for copper and hence elemental contrast is enhanced due to anomalous scattering in the copper layers. Reflectivity measurements were obtained using a two-circle diffractometer with independently controlled $\theta$ and $2 \theta$ circles to an accuracy of $0.001^{\circ}$ and $0.002^{\circ}$ respectively. A goniometer sample stage was mounted horizontally on the $\theta$ (sample) circle, providing two translations and two arcs. The incident beam was defined by $0.1 \mathrm{~mm}$ vertical slits and $10 \mathrm{~mm}$ horizontal slits. The reflected beam was collimated using two pairs of vertical slits set to $0.1 \mathrm{~mm}$, the scatter slits being placed $20 \mathrm{~cm}$ from the sample and the resolution slits at $70 \mathrm{~cm}$. The collimated beam had a Gaussian profile with a full width half maximum of $0.008^{\circ}$. Here we report the results of $\theta-2 \theta$ and offset $\omega$ scans of the samples.

\section{Results and Discussion}

The $\theta-2 \theta$ reflectivity scans for the four samples are shown in Figure 1 as the upper curve in each case, curve (a) being the lowest power film ( $3.65 \mathrm{~W}$ ) and (d) being the highest $(6.35 \mathrm{~W})$. The abscissa is the $2 \theta$ angle and the ordinate the common log of the reflectivity. It is necessary to use a log scale as the reflectivity from a smooth surface falls of as $\theta^{4}$ and more 
quickly from a rough surface. The scans show several interesting features. Firstly, all the scans display Kiessig fringes between 1 and 2 degrees, which originate from multiple beam interference (MBI) caused by strong reflection at the top air interface and the bottom Si interface. The existence of these fringes and the envelope of the reflectivity decay indicate that the top surface of the multilayer stack is indeed very smooth. Secondly, the curves display Bragg reflections originating from the layer spacing of the bilayers, with in some cases the second order being apparent although unexpected for Co:Cu thicknesses of $1: 1$. The presence of the Bragg peaks indicates a well-defined bilayer structure, though the Bragg peak becomes systematically weaker as the deposition power increases. This trend indicates that the bilayer structure becomes less well defined with increasing pulse energy. The weakness of higher order peaks is a sign that the interfaces are not mathematically planar. The third structure visible is a low frequency oscillation in reflectivity originating from MBI due to scattering from the interface on either side of the surface oxide.

To obtain a quantitative analysis of the GIXR curves the data were simulated using the XREALM [9] code, which is based on the recursive scheme of Parratt [10]. The best fit to the data using this code is shown in Figure 1 as the lower of the two curves in each panel. The curve has been displaced from the data for clarity. This fit is sensitive to the thicknesses of all layers and the roughnesses of all interfaces. A summary of the main results is given in Table 1 . The first thing to note is the total film 
thickness. The films are all of similar though not identical thickness showing that the empirical attempt to adjust the pulse per layer to compensate for the differing power regimes has at least partially worked. The second point to notice is the weak increasing trend in interface width with increasing deposition power. Whilst the fit to the whole reflectivity envelope gives a less clear cut variation in interface width than might be expected from consideration of the Bragg peaks alone, it is clear the film grown with highest power has interfaces that are significantly broader than that with the lowest power. The films grown at 3.65, 4.55 and 5.45 W, the mean interface roughnesses could be comparable within experimental error. However, for the film grown at $6.35 \mathrm{~W}$, the interface width at least doubles compared with the $3.65 \mathrm{~W}$ film. The mean width of $11 \AA$ is comparable with half of a single layer size (around $20 \AA$ ), meaning that it becomes difficult to think of the layers as individual. The top surface roughness of the films is $9 \pm 1 \AA$ in all cases, so the increased width of the interfaces in the multilayer deposited with high power is unlikely to be statistical roughness in the interface, which would accumulate. A more probable explanation is that there is intermixing between $\mathrm{Co}$ and $\mathrm{Cu}$ which has reduced the contrast of the interface. These two effects are indistinguishable in the XREALM simulation.

To investigate the vertical correlation of the interface widths, a series of offset $\theta-2 \theta$ were performed. In these scans, the sample was offset from the specular by an angle $\Delta \omega$ to evaluate the diffuse intensity contribution. The replication of the Bragg peak in these scans is a sign 
that there is a vertical correlation in the interface roughness, that the roughness of subsequent interfaces is conformal with lower ones. This effect is most striking in the multilayer deposited with low power, indicating that the reduced intermixing comes with a price of lack of suppression of conformal roughness.

Further information about the microstructure of the films was obtained by the use of microscopy. Figure 3 show a scanning electron micrograph of the as-deposited films. The image shows droplets with dimensions up to $5 \mu \mathrm{m}$ across as is usual with PLD. The embedding of droplets into magnetic multilayers could be a serious problem if they are of a ferromagnetic element as they could provide magnetic bridging that would result in entirely ferromagnetic coupling. However, Auger electron spectroscopy of the droplets indicates that they are mostly $\mathrm{Cu}$.

\section{Summary}

The influence of LASER power on the microstructure of $\mathrm{Co} / \mathrm{Cu}$ multilayers has been investigated using GIXR. It is found that increasing LASER power results in increasing interface width within the films, probably a result of intermixing. Offset scans show that there is a significant fraction of conformal roughness within the layer stack. Auger electron spectroscopy indicates that the droplets created by the ablation process are primarily of $\mathrm{Cu}$. 


\section{Acknowledgements}

The authors would like to acknowledge Polaron-CVT, the DTI, and EPSRC for the support of this project, and Daresbury Laboratory for the provision of SRS beamtime. MSA would also like to acknowledge the Government of Oman for the provision of a Scholarship.

\section{References}

[1] S. S. P. Parkin, R. Bhadra and K. P. Roche, Phys. Rev. Lett. 662152 (1991)

[2] M. Jacob, G. Reiss, H. Bruckl, and H. Hoffman, Phys. Rev. B 4611208 (1992)

[3] A. R. Modak, D. J. Smith and S. S. P. Parkin, Phys. Rev. B 504232 (1994)

[4] D. E. Joyce, C. A. Faunce, P. J. Grundy, B. D. Fulthorpe, T. P. A. Hase, I. Pape and B. K. Tanner, Phys. Rev. B 585594 (1998)

[5] E. E. Fullerton, D. M. Kelly, J. Guimpel, I. K. Schuller and Y. Bruynseraede, Phys. Rev. Lett. 68859 (1992)

[6] N. D. Telling, S. J. Guilfoyle, D. R. Lovett, C. C. Tang, M. D. Crapper and M. Petty, J. Phys. D: Appl. Phys. 31472 (1998)

[7] H Mai and W Pompe, Applied Surface Science 54215 (1992)

[8] C. C. Tang, S. P. Collins, B. M. Murphy, N. D. Telling, R. A. Wogelius, S. J. Teat, Rev. Sci. Instr. 691224 (1998)

[9] N. D. Telling, M. D. Crapper, D. R. Lovett, S. J. Guilfoyle,C. C. Tang and M. Petty Thin Solid Films 317278 (1998).

[10] L. G. Parratt, Phys. Rev. 95359 (1954). 


\section{Figure Captions}

Figure 1: Grazing incidence X-ray reflectivity $\theta-2 \theta$ measurements of Cu/Co multilayers deposited by PLD with varying LASER powers. Panel (a) is for a power of $3.65 \mathrm{~W}$, (b) $4.55 \mathrm{~W}$, (c) $5.45 \mathrm{~W}$ and (d) $6.35 \mathrm{~W}$. In each case the upper case is the raw data and the lower curve is the best fit, which has been displaced for clarity.

Figure 2: Grazing incidence X-ray reflectivity offset scans of $\mathrm{Cu} / \mathrm{Co}$ multilayers deposited by PLD with varying LASER powers. Panel (a) is for a power of $3.65 \mathrm{~W}$, (b) $4.55 \mathrm{~W}$, (c) $5.45 \mathrm{~W}$ and (d) $6.35 \mathrm{~W}$. The lower curve is for $0^{\circ}$ offset and for the higher curves, the sample is offset from the $\theta-2 \theta$ end by an angle $\Delta \omega$ in increasing steps of $0.05^{\circ}$.

Figure 3: A scanning electron micrograph of the PLD deposited films. The micrograph is $150 \mu \mathrm{m} \times 150 \mu \mathrm{m}$. 
Table 1: Width of $\mathrm{Cu} / \mathrm{Co}$ interfaces measured by grazing incidence X-ray reflectivity (GIXR) with LASER power.

\begin{tabular}{|c|c|c|}
\hline $\begin{array}{c}\text { LASER Power at } \\
10 \mathrm{~Hz}\end{array}$ & $\begin{array}{c}\text { Total thickness } \\
\text { of multilayer } \\
\text { measured by GIXR }\end{array}$ & $\begin{array}{c}\text { Effective width } \\
\text { of Co/Cu interfaces } \\
\text { measured by GIXR } \\
( \pm 1 \AA)\end{array}$ \\
\hline $3.65 \mathrm{~W}$ & $88 \mathrm{~nm}$ & $5 \AA$ \\
\hline $4.55 \mathrm{~W}$ & $86 \mathrm{~nm}$ & $7.5 \AA$ \\
\hline $5.45 \mathrm{~W}$ & $99 \mathrm{~nm}$ & $6 \AA$ \\
\hline $6.35 \mathrm{~W}$ & $99 \mathrm{~nm}$ & $11 \AA$ \\
\hline
\end{tabular}


Figure 1

(a)

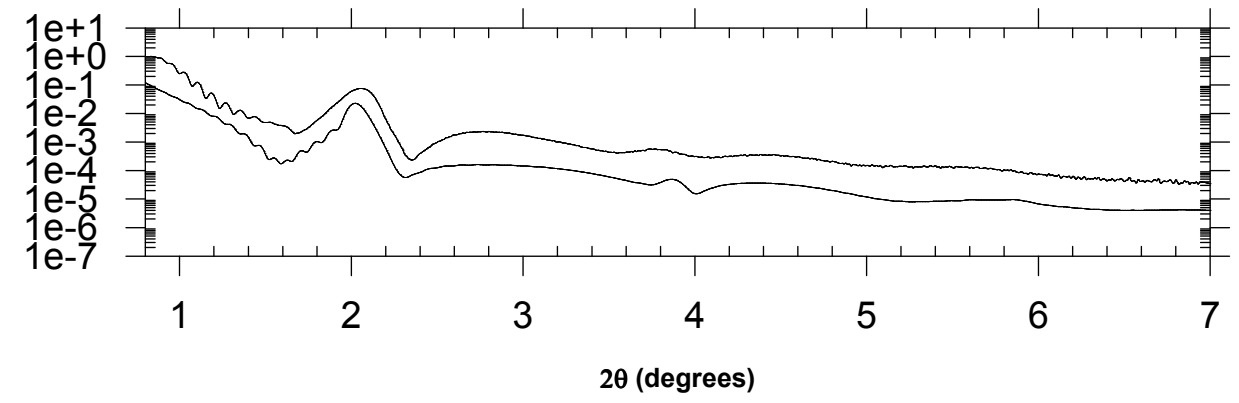

(b)

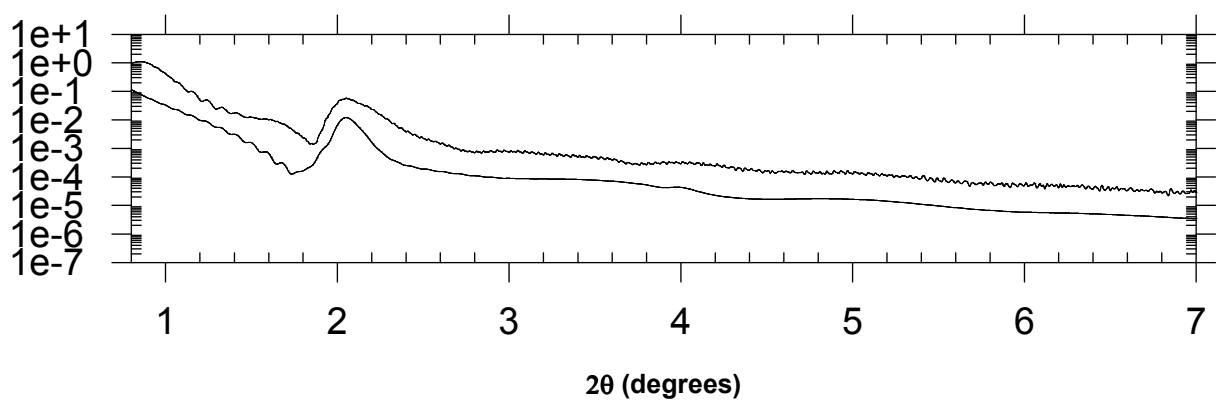

(c)

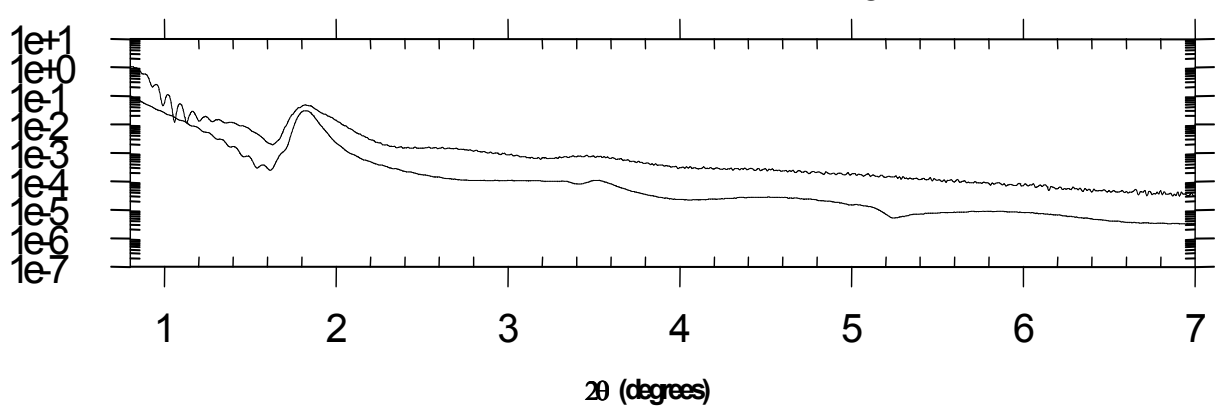

(d)

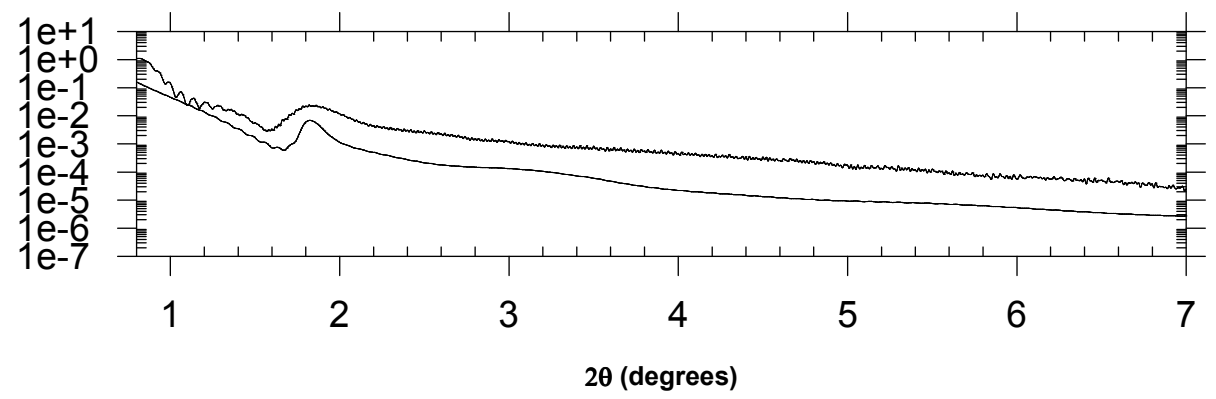


Figure 2

(a)

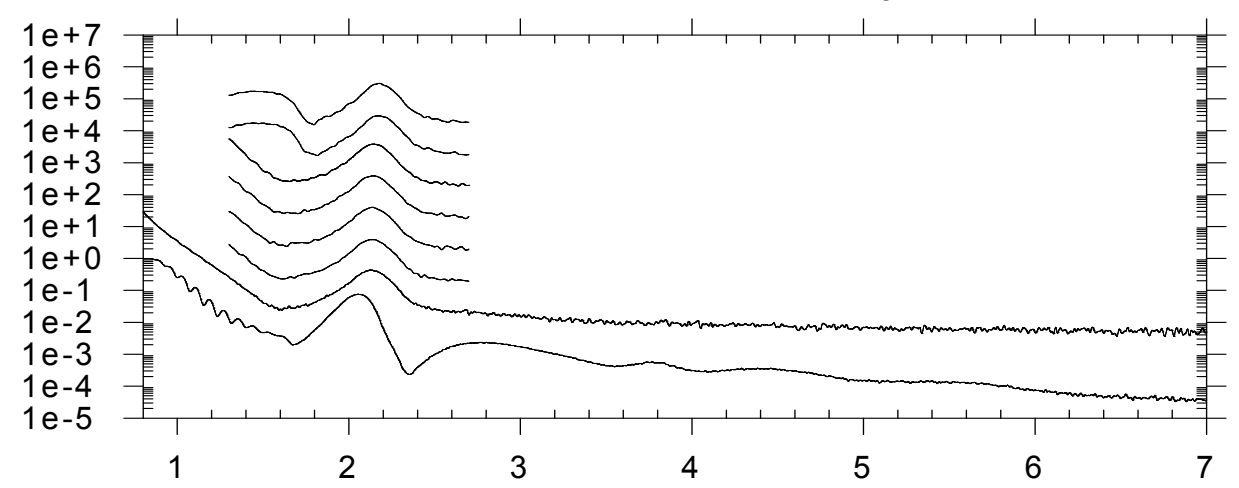

(b)

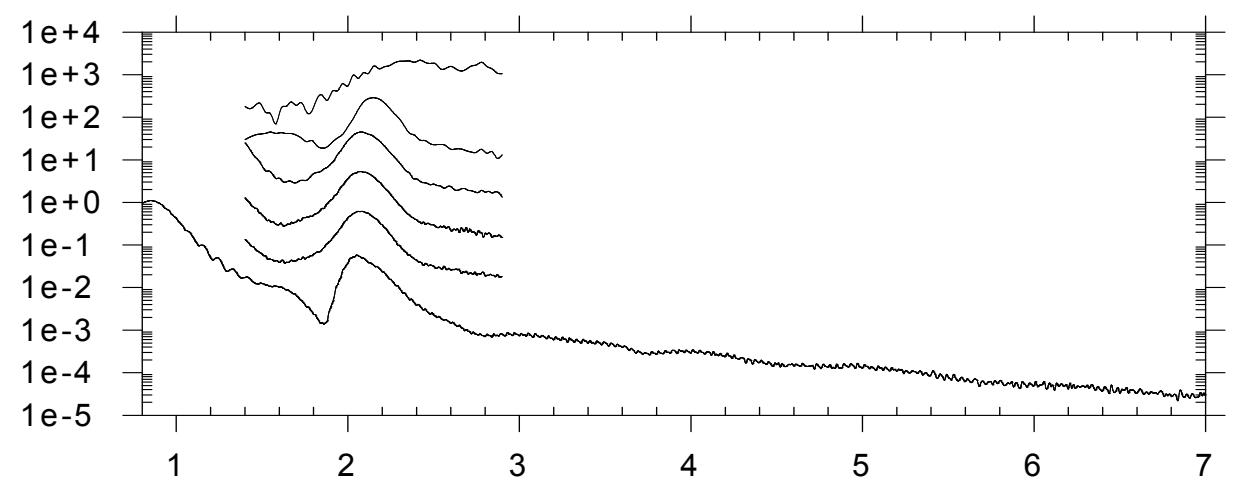

(c)

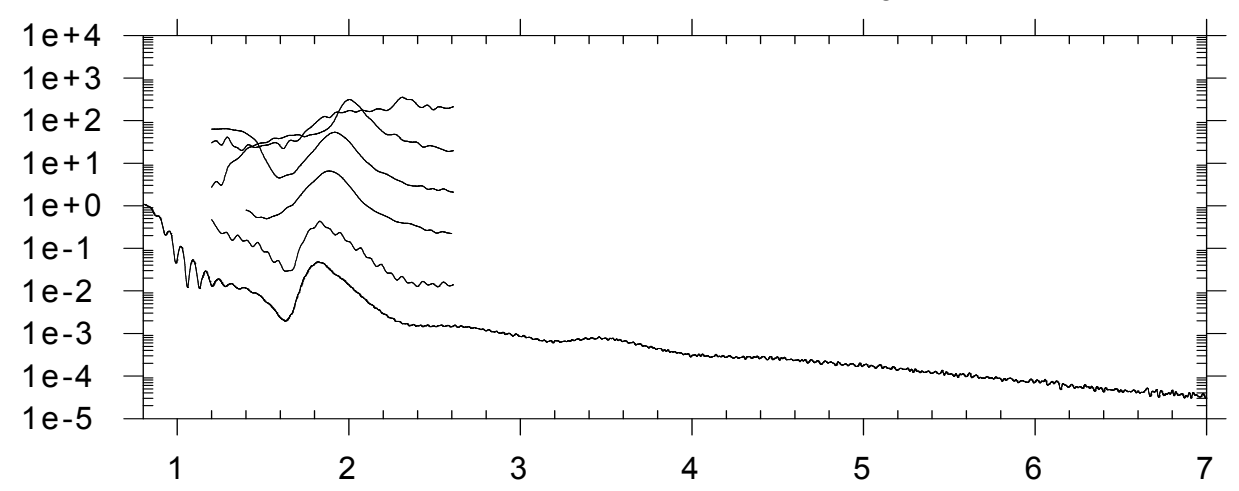

(d)

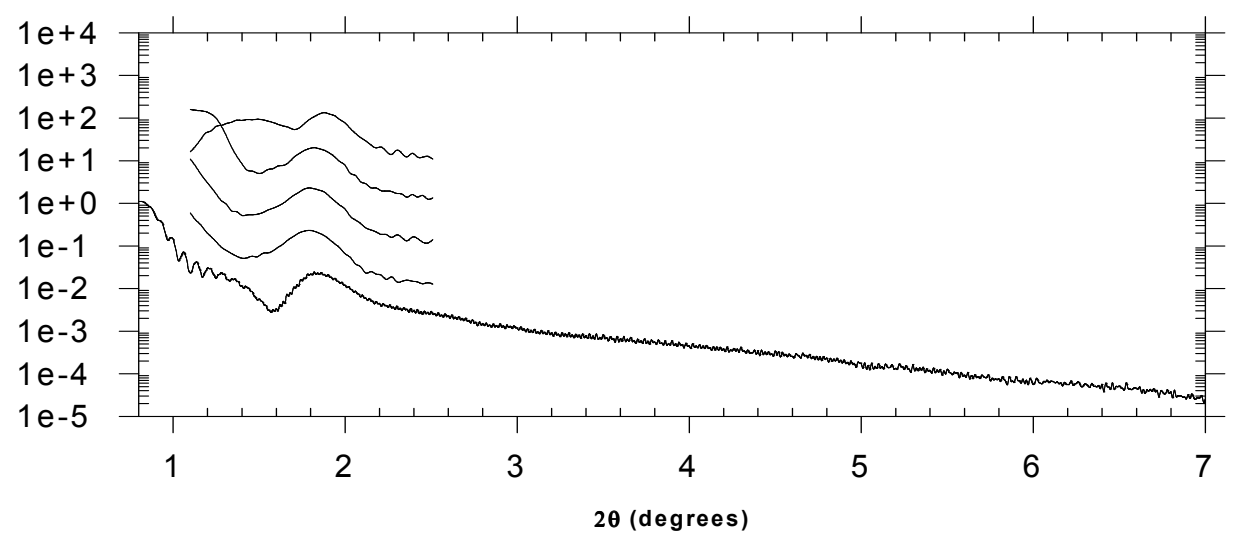


Figure 3

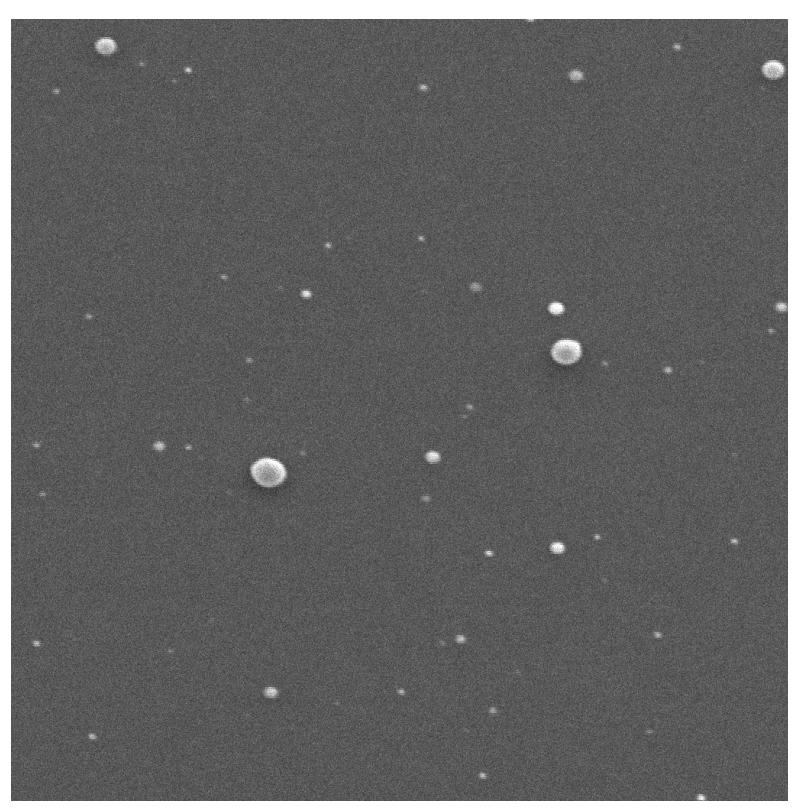

